\title{
Vínculos entre la política de incentivo a la demanda de tierra y vivienda e integración urbana en áreas de crecimiento urbano extensivo. El caso del PRO.CRE.AR en La Plata
}

\author{
Links between the policy of incentive to the demand for land \\ and housing and urban integration in areas of extensive \\ urban growth. The case of PRO.CRE.AR in La Plata
}

María Eugenia Rodríguez Daneri

\section{Resumen}

La presente investigación tiene como propósito analizar el modo en que la política habitacional de incentivo a la demanda impulsada por PRO.CRE. AR incide en los procesos de expansión urbana, analizando los rasgos principales de la integración urbana: aspectos urbanos, ambientales y normativos. Los avances del trabajo permitieron reconocer que uno de los factores que tiene peso significativo es la localización del Programa en áreas no urbanas, como también en aquellas áreas que siendo urbanas, tienen grandes déficit de servicios y equipamientos públicos. Ello da cuenta de la dificultad que tiene el municipalidad para gestionar el acceso al suelo en condiciones urbanas de calidad, en una ciudad que se expande insustentablemente y donde el problema de la tierra es constante.

Palabras clave: política habitacional; integración urbana; expansión urbana; instrumentos; La Plata.

\begin{abstract}
This research aims to analyze the way in which the housing policy of incentive to the demand stimulated by PRO.CRE.AR influences urban expansion processes, examining the main features of urban integration: urban, environmental and regulatory issues. It was possible to notice that one of the most significant factors is the location of the Program in non-urban areas, as well as in urban areas that still have large deficits of public services and facilities. This accounts for the difficulty that the municipal government has in managing access to land in quality urban conditions in a city that expands unsustainably and where the land problem is constant.
\end{abstract}

Keywords: housing policy; urban integration; urban sprawl; instruments; La Plata. 


\section{Introducción}

La presente investigación ${ }^{1}$ tiene como propósito analizar las relaciones de la creciente política de incentivo a la demanda habitacional (Programa Crédito Argentino del Bicentenario - PRO.CRE.AR) con el crecimiento urbano extensivo en el Partido de La Plata. En particular, se propone reflexionar sobre las condiciones de integración urbana emergentes de dicho programa.

Esta investigación se enmarca en un trabajo de investigación mayor que estudia los procesos de expansión urbana, sus características diferenciales y la evaluación de la incidencia de las políticas de ordenamiento territorial, y de tierra y vivienda implementadas por los gobiernos locales en distintos municipios de la Provincia de Buenos Aires. El mencionado estudio permitió verificar que en los procesos de expansión urbana los usos dominantes han sido los residenciales, conducidos en gran medida por el sector privado, pero también por una importante acción de las políticas públicas, en particular las políticas de tierra y vivienda y de producción de infraestructuras (Rocca et al., 2010).

Si bien la normativa provincial vinculada al ordenamiento territorial (Decreto Ley $n^{\circ}$ 8912/1977) establece un marco para regular el crecimiento urbano, implantando estándares de toda nueva subdivisión del suelo, y estableciendo las formas de completamiento urbano de acuerdo a necesidades de incremento poblacional y dotación de infraestructuras, la creciente necesidad de suelo y el desarrollo de mercados inmobiliarios especulativos que encarecen el precio del suelo, promueven la expansión física y extensiva de la ciudad. (Rocca et al., 2010).

La tendencia hacia la expansión en áreas periféricas sobrevaluadas, pero al mismo tiempo carentes de servicios, evidencia la desigualdad de acceso a los servicios urbanos y equipamientos públicos que, a su vez, está vinculada a asuntos tales como la seguridad de tenencia, la pobreza, la exclusión y fragmentación social, el riesgo de inundaciones, la expulsión de actividades agropecuarias periurbanas, entre otros procesos. En consecuencia, puede deducirse que las políticas públicas resultan insuficientes para dar cuenta de las múltiples demandas que posee la población de espacios para la reproducción y producción social.

En este contexto, el artículo explora el modo en que la política habitacional de fomento a la demanda, impulsada por el PRO. CRE.AR, incide en los procesos de expansión urbana analizando los rasgos principales de la integración urbana, es decir aspectos urbanos, ambientales y normativos.

El estudio se estructura en tres partes, en la primera se presenta el marco teórico- metodológico donde se desarrollan los principales conceptos que vinculan la problemática del crecimiento extensivo con las políticas urbanas, instrumentos y objetivos de intervención que permiten identificar los componentes para el desarrollo del estudio de caso. En la segunda se abordan los principales ejes que hacen a la integración urbana, poniendo a prueba dos tipos de iniciativas emergentes del PRO.CRE.AR. En la última se exponen las conclusiones del estudio, donde se reflexiona sobre los factores que afectan directamente a la integración urbana del caso de estudio. 


\section{Crecimiento extensivo: políticas urbanas, instrumentos y objetivos de integración urbana}

Se partió de un marco teórico general que permitió interpretar los procesos actuales de crecimiento urbano extensivo en Latinoamérica y sus vínculos con la planificación y gestión urbana, y los procesos socio-territoriales subyacentes. En articulación con el anterior, la exploración se basó en un marco teórico sustantivo que profundiza en las relaciones entre el acceso al suelo urbano y los distintos instrumentos de la política urbana.

\section{Crecimiento urbano extensivo: teoría sobre la realidad latinoamericana}

La expansión urbana es tanto un producto como un proceso que expresa el crecimiento urbano por extensión con modalidades específicas históricamente determinadas por el contexto social, económico y político en que se materializa. Dichas modalidades se expresan territorialmente en la estructura de la ciudad, así como en las nuevas relaciones entre la ciudad y su entorno, entre distintas ciudades, y el territorio en sus diferentes escalas.

Para abordar esta problemática la investigación se enmarcó en las coincidencias teóricas generales que pueden trazarse entre distintos autores que relacionan la tendencia a la urbanización global con las profundas transformaciones en las formas de producción y configuración de las ciudades en el contexto capitalista actual, donde la expansión descontrolada y los cambios en la demanda de suelo, definen gran parte de la problemática urbana actual. Así, el modelo resultante justifica la necesidad del manejo del crecimiento urbano a partir de políticas públicas planificadas e integrales como un desafío en el mundo entero.

Está confirmada la tendencia mundial hacia la urbanización y la forma insustentable en que esta viene desarrollándose. A nivel global, el modelo dominante es la metropolización expandida y descontrolada, con configuraciones urbanas difusas y extendidas y agudización de los problemas sociales y ambientales con patrones de segregación y exclusividad del crecimiento (Aguilar, 2006; De Mattos, 2010). La expansión urbana en la mayoría de las regiones metropolitanas latinoamericanas muestra un panorama de pobreza, informalidad e ilegalidad de los patrones de uso del suelo, y una ausencia de infraestructura, equipamientos y servicios básicos (Lungo, 2007) como correlato de las dificultades del acceso a la tierra y vivienda urbana.

Sobre estos procesos ha tenido una gran actuación el crecimiento incontenible de la inversión inmobiliaria, con procesos de creciente mercantilización del desarrollo urbano, en los que la plusvalía urbana se ha transformado en el criterio urbanístico dominante (De Mattos, 2010). Ello justifica la necesidad de comprender mejor la expansión de las ciudades en países en desarrollo y asegurar, a través de planes y políticas de gestión de la expansión urbana, el desarrollo ordenado de las ciudades para hacerlas más 
eficientes, equitativas y sostenibles (Shlomo y otros, 2012). En el mismo sentido se necesita comprender mejor las políticas urbanas, instrumentos y objetivos de intervención sobre las áreas de crecimiento extensivo para evaluar su contribución a la integración urbana.

En línea con lo anterior, resultan fundantes los estudios de Aguilar (2002), De Mattos (1999) y Cicolella (1999) porque a través del análisis del crecimiento de las grandes ciudades confirman que los más importantes cambios se dan en los espacios de crecimiento extensivo con patrones de dispersión urbana que incorpora progresivamente pueblos y periferias rurales dentro de un sistema metropolitano cada vez más amplio y complejo.

Por su parte, el trabajo de Rocca (2013) es particularmente significativo porque en la exploración comparativa de las características de los procesos de expansión urbana en ciudades de distinto tamaño y situación territorial dentro de la Provincia de Buenos Aires, analiza sus causas y vínculos con la política territorial durante los últimos 35 años. Este trabajo comprueba que existe una consolidación de áreas de expansión urbana con altos contrastes sociales y espaciales, y en términos cuantitativos, se verifica un crecimiento acelerado y extensivo de las áreas urbanizadas en relación al incremento poblacional y de la cantidad de viviendas.

Otro aspecto interesante en la investigación que desarrolla Rocca (2013) es el análisis de las normativas de zonificación municipal - instrumento generalizado en el territorio bonaerense - donde se verifica que al no tener Planes que las enmarquen resultan indeterminas en cuanto a principios y lineamientos de las áreas previstas para la expansión urbana. Esto, sumado a la falta de proyectos y/o mecanismos de gestión que viabilicen desde una modalidad proactiva, disminuye las posibilidades de la regulación municipal dejando a criterio de las iniciativas privadas $u$ otros organismos sectoriales la conducción del proceso.

Asimismo, también verifica la conjugación de factores estructurantes y específicos en la impulsión de la expansión urbana extensiva. Pueden reconocerse como causas estructurales del proceso, no solo las importantes transformaciones económicas y productivas producidas en los centros urbanos, sino también los altos déficits de tierra y vivienda accesibles para las clases medias y bajas, las demandas de nuevos estándares habitacionales para las clases medias y altas, conjugados con políticas públicas nacionales, provinciales y locales que favorecen los procesos expansivos.

Cabe precisar que, los factores específicos que articuladamente con los anteriores, han contribuido a moldear la situación en cada ciudad, se vinculan con transformaciones en los perfiles productivos, las pertenencias regionales, la dotación de recursos naturales, sus condiciones de accesibilidad, entre otras cuestiones.

Para comprender la integralidad de la problemática de crecimiento urbano extendido es necesario entender las dificultades de acceso a la ciudad, porque el mercado legal del suelo resulta inaccesible a la población de bajos ingresos. Sobre este proceso, tiene una alta incidencia la regulación del suelo porque no implica una intervención significativa en el mercado que favorezca el acceso a la tierra de los sectores sociales de menores ingresos 
(Clichevsky, 2003). Las posibilidades que tiene la población de acceder a vivir en una determinada área urbana se relaciona con las condiciones de pobreza y el funcionamiento del mercado legal de tierras y vivienda. Según la autora el mercado legal del suelo resulta inaccesible a la población de bajos ingresos; y las políticas estatales sobre regulación del mercado privado del suelo han influido decisivamente en el acceso porque no implicaron una intervención significativa en el mercado tendiente a favorecer el acceso a la tierra de los sectores sociales de menores ingresos.

Por su parte, las políticas de Tierra y Vivienda, mayormente generadas desde los niveles de gobierno intermedio y superior, no han sido articuladas adecuadamente con los gobiernos locales, considerado su incidencia en la conformación de áreas de expansión urbana, contribuyendo a la dispersión y fragmentación espacial y social. Estas políticas, actuando con objetivos sectoriales, impulsaron el crecimiento hacia áreas no previstas, y presionaron sobre otros usos de la tierra de menor relevancia económica, como pueden ser los espacios verdes abiertos o los agropecuarios (Lancioni et al., 2012).

\section{Instrumentos de tierra y vivienda en el contexto de las políticas urbanas}

El crecimiento urbano extensivo requiere un abordaje multidimensional acorde a la complejidad de la problemática. En este sentido resulta útil la propuesta de Antonio
Azuela (2007), donde propone reivindicar el valor social del suelo en las políticas en torno al mercado de suelo urbano que reconozcan el valor estratégico de la tierra y las características particulares del funcionamiento de sus mercados, para impulsar un uso sostenible del suelo al incorporar objetivos sociales y ambientales y beneficiar a los segmentos más vulnerables de la población urbana. Para ello es imprescindible que el Estado, tanto en el ámbito municipal como el nacional, tenga un rol activo en la promoción del desarrollo urbano a través de políticas e instrumentos integrados que puedan favorecer la integración como objetivo de cualquier acción territorial.

Morales (2005), por su parte, sostiene que dejar la asignación del recurso suelo a los intereses particulares implica una distribución desigual de dicho recurso. La definición del interés público del suelo urbano podría integrar componentes que aseguren que la ciudad sea: equitativa, convivida, integrada al medio natural, y productiva. Por ello la importancia de la regulación del suelo a través de diversos instrumentos.

Distintos autores (Fernández Wagner, 2006; Del Río, 2011) coinciden en que en Argentina, durante las últimas décadas, los principales instrumentos implementados por la política habitacional, con las diferencias que Morales (2005) realiza entre "las de oferta de vivienda construida", y "las de fomento de la demanda", intentaron dar respuesta a esta problemática, pero al no articularse con políticas urbanas también favorecieron el crecimiento extensivo de la ciudad.

Fernández Wagner (2006) señala que se ha centrado el foco en la construcción masiva de viviendas, pero al no existir una 
correspondencia entre la planificación urbana y la disponibilidad de un conjunto de instrumentos organizados en tal sentido, tiene una serie de consecuencias negativas o al menos contradicciones, entre las que destaca la existencia de escasez de suelo de propiedad pública disponible. Se debe enfrentar la problemática de habitar la ciudad de modo complementario con el desarrollo de una regulación del suelo urbano y la planificación en la esfera local.

En la misma línea, Del Río (2011) señala que, en gran medida, este esquema de urbanización es producto de la incapacidad de la gestión urbana para intervenir e introducir mecanismos de regulación de los mercados de suelo urbano; de la connivencia de intereses de actores públicos y privados tendientes a dinamizar el capital inmobiliario; y de la difusión de concepciones urbanísticas excluyentes mediante los estándares normativos o de zonificación, que rara vez asimila las prácticas y procesos populares de producción del hábitat.

Es importante destacar que la mayor oferta residencial desarrollada en Argentina desde 2003 no implicó precisamente mejores condiciones de acceso a la vivienda, en tanto la inversión inmobiliaria se hace como reserva de valor y solo para la población de mayor poder adquisitivo (Baer, 2008).

Por lo tanto, el gobierno no debería ignorar su responsabilidad de adoptar políticas en torno al mercado de suelo urbano que reconozcan el valor estratégico de la tierra y las características particulares del funcionamiento de sus mercados, para impulsar un uso sustentable del suelo al incorporar objetivos sociales y ambientales y beneficiar a los segmentos más vulnerables de la población urbana.

\section{La integración como objetivo}

Lo anteriormente escrito afirmó que la expansión urbana en Latinoamérica se caracteriza por procesos imbricados de fragmentación, dispersión, segregación, desintegración y desigualdad, en términos espaciales, sociales, económicos y políticos.

Los gobiernos enfrentan cotidianamente la necesidad de integrar las poblaciones vulnerables y marginadas en los territorios urbanos. En relación con esto, Sabatini (2008) explica que la segregación de los grupos populares en la periferia de las ciudades tiene impactos urbanos y sociales. Entre los primeros destacan los problemas de accesibilidad y la carencia de servicios y equipamientos en sus lugares de residencia; y entre los segundos, los problemas de desintegración social. Ello, representan formas de empobrecimiento o de degradación social vinculados a las desventajas que conlleva la falta de integración física.

En la misma línea, Vidal Rojas (1997) entiende la fragmentación urbana como un proceso territorial mayor, que se construye a través de tres subprocesos: fragmentación social, fragmentación física y fragmentación simbólica. En cualquiera de los casos supone la independencia de las partes (fragmentos) en relación al todo (sistema urbano). El abordaje de la cuestión de la fragmentación urbana reconoce fundamentalmente dos líneas de análisis aunque con diferentes matices en cada una de ellas, por un lado, aquella que se 
halla ligada a procesos de desigualdad social y barreras materiales y/o inmateriales; por el otro, la que se relaciona con las discontinuidades en el proceso de expansión urbana a respecto de la trama producto de los procesos de metropolización (Valdes, 2001).

En la búsqueda de operativizar estos conceptos que definen procesos involucrados en los procesos de expansión Del Río (2008) señala que la calidad urbana es la cualificación del espacio urbano que expresa la articulación compleja entre accesibilidad a las infraestructuras, conectividad al trasporte público y proximidad a la centralidad urbana. Así que sistematiza una serie de variables que tiene por objetivo caracterizar las condiciones de calidad urbana. Se refiere a la centralidad urbana la relación entre accesibilidad/ conectividad, consolidación urbana de servicios en la vía pública, conexión a servicios sanitarios básicos, calidad de ocupación de la vivienda y calidad constructiva de la vivienda.

También propone analizar variables a nivel socioeconómico para determinar la posición relativa de las tipologías analizadas en la estructura socioespacial. Finalmente, se identifica la distancia al borde de la mancha urbana para analizar la inserción de conjuntos habitacionales sobre áreas rurales o de borde urbano.

En términos generales la integración urbana expresa como se relacionan los distintos componentes físicos, ambientales, y socioeconómicos de la ciudad y a su vez actúa como un indicador que permite aproximar el nivel de calidad urbana de una ciudad, siendo la integración social un proceso vinculado y condicionado por la misma.

\section{Metodología}

Él marco teórico sirvió para la construcción de la metodología, del mismo modo los distintos estudios que analizan y evalúan soluciones habitacionales. Esto permitió llegar a la conclusión que la integración urbana puede entenderse a partir de la relación interrelación de distintos ejes. En función de esto, se construyó una matriz de análisis (Cuadro 1), de carácter exploratorio, donde se analizaron distintos ejes que permitieron reflexionar sobre los factores que determinan la integración urbana de las localizaciones del PRO.CRE.AR.

En la matriz, la primera columna representa los ejes de análisis, que son aquellas que a partir de su interrelación definen la integración urbana. En la segunda columna se detallan las variables que se evalúan de cada eje de análisis. La tercera columna está compuesta por el tratamiento del dato, por ejemplo: distancia en metros hasta la centralidad más cercana, en una escala del 1 al 4 expresando de izquierda a derecha mejor condición a mayor déficit.

Para la producción cartográfica y tablas síntesis se ensambló un Sistema de Información Geográfica (SIG) y se relacionó con técnicas de análisis espacial para relacionar la localización del Programa con las variables de análisis, con el fin de analizar los rasgos principales de la integración urbana que emerge del Programa. En algunos casos se utilizaron medidas tradicionales de distancia generando buffers, en otros casos se ajustó una medición distinta de acuerdo a las necesidades de cada variable. 
Cuadro 1 - Matriz de análisis

\begin{tabular}{|c|c|c|c|c|c|}
\hline \multirow{2}{*}{ Eje } & \multirow{2}{*}{ Variable } & \multicolumn{4}{|c|}{ Tratamiento del dato } \\
\hline & & 1 & 2 & 3 & 4 \\
\hline Ambiental & Riesgo hídrico & Sin riesgo & Muy bajo / bajo & Medio & Alto \\
\hline \multirow{5}{*}{ Urbano } & Servicios básicos & Con agua y cloaca & Con agua & Con cloaca & Sin servicios \\
\hline & $\begin{array}{l}\text { Equipamiento } \\
\text { salud }\end{array}$ & A menos de 300 & Entre 300 y 600 & Entre 600 y 900 & A más de 900 \\
\hline & $\begin{array}{l}\text { Equipamiento } \\
\text { educativo }\end{array}$ & A menos de 600 & Entre 600 y 900 & Entre 900 y 1.200 & A más de 1.200 \\
\hline & Subcentralidades & A menos de 2.200 & Entre 2.000 e 3.000 & Entre 3.000 y 4.000 & A más de 4.000 \\
\hline & Transporte público & A menos de 300 & Entre 300 y 600 & Entre 600 y 900 & A más de 900 \\
\hline Regulatorio & $\begin{array}{l}\text { Zonificación de } \\
\text { usos de suelo }\end{array}$ & Urbana & Complement. & Rural & Rural \\
\hline
\end{tabular}

Fuente: elaboración propia.

A continuación se repasan los criterios de definición de cada uno de los ejes de análisis que componen la matriz:

- El eje ambiental refiere a cuáles son las condiciones ambientales en que se localiza el Programa. Es decir, interesa conocer si el Programa está localizado en áreas con riesgo de inundaciones. Para ello, la variable que se evalúa es:

1) Riesgo Hídrico. Para analizar la localización en relación al riesgo hídrico se toma como base la cartografía elaborada por el Centro de Investigaciones en Suelos y Agua de Uso Agropecuario (CISAUA) en el marco de un estudio de Riesgo Hídrico en el Partido de La Plata. Para construir el mapa de Riesgo Natural de Inundación, dicho estudio se basó en las distintas inundaciones (2002 y 2008) que afectaron al partido y que demuestran el colapso del sistema de desagües tanto del casco urbano como de su periferia.
- El eje urbano hace referencia a la distribución de bienes y servicios. En este eje es importante evaluar si el Programa está ubicado cercano a centralidades o subcentralidades urbanas, a equipamientos públicos, si cuenta con cobertura de servicios básicos, entre otras cosas. Para ello las variables que se analizan son:

2) Servicios básicos agua y cloaca. El análisis de la cobertura se realiza a partir de los datos obtenidos de ABSA respecto de los servicios de agua y cloaca e identificando si la localización del Programa se encuentra dentro de estas áreas servidas.

3 y 4) Equipamientos públicos de Salud ${ }^{2}$ y de Educación. ${ }^{3}$ El análisis de los equipamientos públicos implica indagar la localización del Programa respecto de la distribución de los equipamientos públicos. Es decir, cuales son las distancias que se deben recorrer para poder acceder a ellos mediante la generación de buffers que miden la proximidad directa. 
5) Centralidades Urbanas. El análisis de centralidad se realiza a partir de comprender que ésta es producto de atracción de flujos, espacios de consumo, concentración de actividades comerciales y financieras. Para ello se identifican los subcentros urbanos y subcentros de servicios establecidos en el Código de Ordenamiento Urbano ${ }^{4}$ vigente; y a través de la generación de distintos buffers se evaluar la proximidad directa.

6) Transporte público. ${ }^{5}$ Las redes de transporte cumplen un papel fundamental para facilitar las interrelaciones en la sociedad, y en particular en este estudio son fundamentales para facilitar la conectividad de las otras variables. Para ello se calculan cuáles son las distancias que se deben recorrer para poder acceder a ellos mediante la generación de buffers que miden la proximidad directa.

- Por último, el eje regulatorio refiere a cuál es la inserción del Programa en relación a las áreas y usos normados según el Decreto-Ley 8912/77 y el Código de Ordenamiento Urbano del partido de La Plata, en particular con aquellas áreas que no son urbanas. La variable que se analiza es:

7) Regulación de usos de suelo. El análisis de la regulación se realiza a partir de identificar cuáles son las zonas destinadas al uso residencial e identificar qué relación tienen éstas con la localización del Programa. Entendiendo que las áreas apropiadas para la localización del Programa son las urbanas.

Como síntesis, del análisis de la localización se identifica:

- los factores que afectan la integración urbana del caso de estudio. A partir de identificar los ejes que tienen mayor incidencia en la configuración de las mayores criticidades.
Este análisis resulta fundamental para poder realizar la evaluación del siguiente punto:

- los sectores urbanos más críticos del partido. Se realizara a partir de la evaluación y relación de las distintas variables; para ello se realizará un cruce entre las mismas, que se encuentren en los últimos dos casilleros de la matriz, los cuales indican las situaciones más críticas. Es decir, todas aquellas localizaciones que cuenten con al menos 3 variables en la escala 3 y 4 de la matriz se georreferenciaran en GIS para comprobar si el conjunto de localizaciones define sectores dentro de la ciudad.

\section{Relaciones entre el PRO.CRE.AR e integración urbana en áreas de crecimiento urbano extensivo}

El Partido de La Plata forma parte de la RMBA, en particular de su litoral sur, es Capital de la Provincia de Buenos Aires, por lo tanto, sede de gobierno y de la administración de los tres poderes provinciales.

Desde fines de los 70, el municipio de La Plata transita un proceso de planificación urbana basado en una serie de instrumentos normativos municipales, enmarcados en la legislación provincial, con el propósito ordenar el crecimiento urbano. Si bien desde esa fecha a la actualidad cuatro han sido las ordenanzas municipales, que tomaron la zonificación del territorio como instrumento de ordenamiento urbano territorial, ninguna de ellas fue concebida en el marco de un plan que defina el modelo urbano y territorial, ni las estrategias, 
programas y proyectos que en conjunto contribuyan a implementar un proyecto de ciudad consensuado por los distintos actores del Partido de La Plata (Rocca y Ríos, 2010).

El trabajo que realizaron Rocca y Ríos sobre los procesos de expansión urbana en el Partido de La Plata permitió verificar que la ciudad creció por extensión en todas sus direcciones. Inicialmente en las áreas de borde y en distintos asentamientos ubicados sobre las principales vías de acceso, posteriormente la mancha urbana se extendió sobre corredores viales con bajas densidades uniendo los distintos asentamientos originados en torno a espacios ferroviarios, y presentando interrupciones básicamente por la presencia de planicies de inundación de arroyos, cavas, grandes equipamientos y espacios destinados a la producción, y terrenos vacíos expectantes.

Por otra parte, la materialización de infraestructuras viales ejecutadas en la década de los 90, pertenecientes al sistema de autopistas metropolitanas, dio lugar a nuevas vinculaciones con la ciudad de Buenos Aires, reforzando la inserción urbana de La Plata en la Región Metropolitana de Buenos Aires, y generando tensiones en el proceso de expansión urbana. Nuevos frentes de urbanización a nivel local en relación a la Auto vía Ruta 2, la Ruta 36 y la Autopista La Plata Buenos Aires.

Se verifica que el crecimiento urbano extensivo ha modificado la configuración de gran parte del territorio conformando distintos sectores urbanos donde se identifican claramente tres ejes de crecimiento: noroeste, sudoeste y sureste; presentando características distintivas desde el punto de vista social y urbano para cada una de las periferias del Partido.

Las tendencias que definen el perfil de la periferia noroeste están vinculadas con una revalorización del suelo urbano que genera expectativas por parte del sector inmobiliario, particularmente en las áreas que han tenido mayor desarrollo económico y que verificaron mejoras en las condiciones de accesibilidad y cualidades ambientales específicas, manteniendo en ese sentido su diferenciación respecto a las restantes periferias. En cambio, las tendencias de dispersión en la periferia sureste (cercanía a Villa Garibaldi) y en la sudoeste (inmediaciones de la R36) están asociadas a sectores de recursos medios que intentan acceder con menores costos a ámbitos en contacto con la naturaleza.

Si bien este fenómeno dio respuesta a nuevas demandas sociales de espacio, particularmente de vivienda, tanto el desarrollo económico sostenido, como la cohesión social y la sustentabilidad ambiental, parecen constituir condiciones difíciles de alcanzar en el marco de este proceso.

Finalmente, las modalidades de apropiación del suelo por parte de sectores de escasos recursos expresadas en asentamientos informales y villas, vinculados a los cursos de agua y espacios ferroviarios, en mayor medida se localizan en las periferias sureste y sudoeste, pero a su vez resulta una tendencia que se incorpora al escenario actual de la periferia noroeste y alerta sobre nuevas condiciones de riesgo hídrico sobre la población.

Esto, pone de manifiesto nuevas tendencias de "la periferia dual, fragmentada social y espacialmente", en ámbitos pobres, degradada, desestructurada; y con espacios 


\section{Foto 1 - Ejes Noroeste, Sudoeste y Sureste, respectivamente}

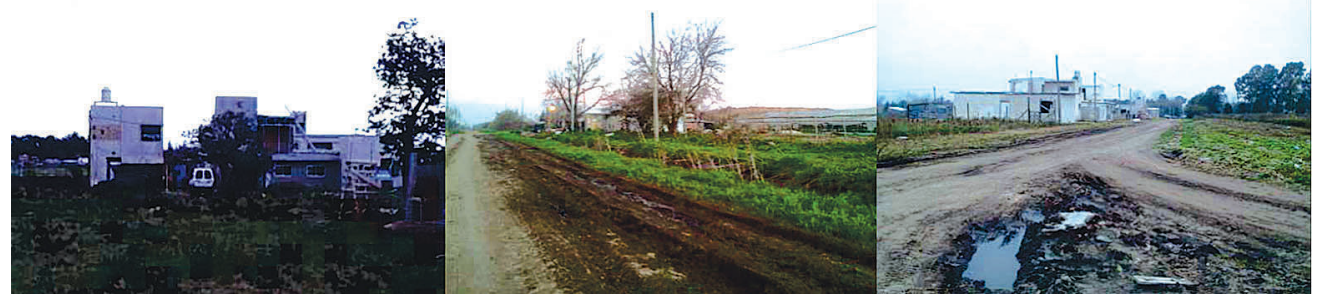

Fuente: Elaboración propia (2014).

"en transición", donde se pronuncian procesos de dispersión urbana, simultáneamente con avances de la urbanización sobre el área complementaria y rural destinada al cinturón hortícolas (Rocca y Ríos, 2010) (Foto 1).

En consecuencia con lo anterior, el avance de la urbanización desencadena un retroceso de las actividades productivas. Tierras que se destinaban a explotaciones primarias intensivas presentan ahora otros usos del suelo propios de un área urbana. Ello genera nuevos loteos y una gran especulación de los agentes inmobiliarios con el fin de obtener el máximo beneficio. Así, la periferia se ve afectada por una dinámica de retención - especulación en el marco de un proceso de suburbanización que progresa principalmente hacia el noroeste tendiendo a conectar la ciudad con Buenos Aires (Frediani, 2010).

Se puede sintetizar, entonces, que la expansión urbana en La Plata se relaciona con los siguientes problemas: avance de la urbanización sobre actividades productivas agropecuarias, y sobre áreas ambientalmente degradadas, dispersión con baja densidad poblacional, incompatibilidad de usos del suelo, aumento del valor de la tierra urbana y no urbana, entre otros. Fenómeno que a escala metropolitana repercuten en un aumento de movimientos residencia-trabajo y en altos déficit de equipamientos e infraestructuras de servicios y comunicación.

\section{El programa analizado}

PRO.CRE.AR Bicentenario es una iniciativa del Gobierno Nacional que proyecta la entrega de 400 mil créditos hipotecarios para la construcción, compra de terreno y construcción, ampliación, terminación y refacción de viviendas, como así también para adquirir aquellas que son construidas por el Programa a través de desarrollos urbanísticos. Tiene como objetivos:

- Atender las necesidades habitacionales de los ciudadanos de todo el territorio nacional, contemplando las diferentes condiciones socioeconómicas y la multiplicidad de situaciones familiares con líneas de crédito para la construcción de viviendas particulares y desarrollos urbanísticos de alta calidad.

- Impulsar la actividad económica a través del incentivo a la construcción de viviendas y su efecto dinamizador.

- Generar empleo en todo el país mediante mano de obra directa e indirecta. 
Sus propósitos son generar desarrollo, trabajo, consumo, y descomprimir el mercado inmobiliario, mejorando las condiciones de quienes no pueden tener acceso mediante el crédito hipotecario o el ahorro personal con destino a la vivienda propia. Este instrumento se implementa en el marco de una política de fuerte intervención pública en la política de vivienda, desarrollada desde el año 2003 a partir de la implementación de los Programas Federales de Vivienda.

Durante muchos años en Argentina, la posibilidad de obtener créditos hipotecarios se vio restringida. En el año 2012, la creación de un fondo fiduciario (PRO.CRE.AR), dio la posibilidad de acceder a la vivienda a través de un crédito con tasas acomodadas en treinta o cuarenta años. Esto generó una demanda enorme que luego fue condicionada por una oferta especulativa que reaccionó como acostumbran los mercados, es decir sacando el máximo nivel de ganancia posible.

Para aplacar este fenómeno cada municipio debía aumentar la oferta de suelo urbano, pero en las diferentes estrategias para llevarlo aparecieron las diferencias. En consecuencia, el Programa ha tenido un impacto distinto en cada Partido; y, como se dijo anteriormente, está directamente relacionado a las capacidades con que cada municipio cuenta para implementar distintos instrumentos de gestión, regulación, etc., teniendo en cuenta ciertos componentes básicos de la urbanización: suelo, servicios básicos, accesibilidad, condiciones urbanoambientales.

En el Partido de La Plata se impulsó el mecanismo que fue denominado "registro de oferentes de terrenos", donde a través de la sanción de la Ordenanza n¹1094/13 se autoriza la re-zonificación de tierras. De esta manera la Ordenanza contempla la formación de un registro de oferentes de tierras, propietarios de hectáreas actualmente indivisibles, por estar zonificadas como rurales o rurales intensivas, y al mismo tiempo se genera un registro de beneficiarios PRO.CRE. $A R$, que son los que podrán comprar estos lotes una vez subdivididos y listos para ser aceptados por el crédito.

Simultáneamente, como una segunda vía de acceso a las tierras, la ordenanza brinda la posibilidad de que grupos de beneficiarios del PRO.CRE.AR compren fracciones indivisas de tierra con destino a la construcción de vivienda (consorcio), para que, una vez garantizados los requisitos que estipula la ordenanza, puedan ingresar esas tierra al registro de oferentes con el proyecto de subdivisión.

Pese a que el Decreto Ley 8.912/77, que regula las urbanizaciones, indica que "las áreas o zonas que se originen como consecuencia de la creación, ampliación o reestructuración de núcleos urbanos y zonas de usos específicos, podrán habilitarse total o parcialmente sólo después que se haya completado la infraestructura y la instalación de los servicios esenciales fijados para el caso, y verificado el normal funcionamiento de los mismos"; la Ordenanza 11094/13 solo exige tendido eléctrico, alumbrado público y apertura de calles transitables, apoyándose en la Ley de Hábitat 14.449, que cuando se trata de re-zonificar parcelas rurales, "Ilama" al artículo 83 del Decreto Ley 8.912/77 que hace referencia a la convalidación por provincia de las ordenanzas municipales que intentan ampliar la zona urbana. 
En este proceso, la tierra aumentó su valor en forma extraordinaria, y el registro de oferentes de terrenos solo quedó limitado por la condición de que el precio máximo de cada lote sea de 150 mil pesos, dejando que esa ganancia excepcional quede en manos del propietario de la tierra, y en muchos de los casos estas tierras no se encuentran dentro de la mancha urbana actual.

Se seleccionaron dos unidades de análisis en correspondencia con dos operatorias llevadas a cabo por el Programa, y desarrolladas en el mismo recorte temporal: primer trimestre del año 2014. Las unidades de análisis seleccionadas son:

La Línea Construcción (Caso 1), destinada a aquellas familias que cuenten con un terreno. En este caso se examinó la información oficial de permisos de obra otorgados en el primer trimestre del año $2014 .{ }^{6}$ Como primer resultado de ello se obtuvo que de los 1.282 permisos de obra aprobados, 437 pertenecen a la Línea Construcción del PRO.CRE.AR.

La Línea Compra de Terreno y Construcción de Vivienda (Caso 2), destinada a familias que aún no disponen de un terreno. Para este caso se utilizaron las diez re-zonificaciones de fracciones indivisas aprobadas por el municipio platense, en el primer trimestre del año 2014, que prevé una oferta de 890 lotes distribuidas en distintos sectores del partido.
Lógicas de localización y análisis de integración urbana del PRO.CRE.AR

\section{a) Eje Ambiental}

1) Riesgo hídrico

Los resultados obtenidos en la especialización del riego hídrico y de los registros PRO.CRE.AR muestran que, si bien la mayoría de los registros de los dos cosos se encuentran en la escala 1 y 2 de la matriz, una considerable parte de los mismos están ubicados en zonas con riesgo, lo que implica no solo un conflicto ambiental sino también un importante conflicto para las familias asentadas en dichos lugares, por estar expuestas no solo a inundaciones (de acuerdo al grado del riesgo) sino también al anegamiento de dichos sectores.

El análisis demuestra que el Programa ha llevado a la ocupación urbana de zonas no aptas, de esta forma gran parte de las planicies de inundación de los arroyos han sido integradas a las áreas urbanas sin ningún tipo de restricción. Ello evidencia la importancia de evaluar, previamente a la aprobación de futuros registros, cuáles son los sectores más convenientes para la localización del Programa en relación al Riesgo; ya que una vez establecidos los mismos se torna muy compleja su reubicación debido al costo económico que esto representa, y más aún el importante costo social que implica trasladar una población ya consolidada. 


\section{b) Eje urbano}

1) Infraestructuras de servicios básicos: Agua y Cloaca

Los resultados en materia de cobertura distan considerablemente de las condiciones que establece el Programa - los lotes deben tener los servicios básicos. La cobertura de infraestructura de servicios en las áreas donde se localizaron los registros representó, en el Caso 1, tener el mayor porcentaje de localizaciones sin ninguno de los dos servicios. Para el Caso 2, la cobertura de servicios resultó aún más crítica ya que ninguno de los registros de esta línea cuenta con los servicios básicos.

El resultado permite observar la desigualdad socio-espacial que subyace en la expansión de la cobertura. Esto da cuenta no solo de las malas condiciones en que se localizan ambas líneas, en particular el Caso 2 , y del impacto que genera en las familias, tanto en lo económico como en lo ambiental, sino también de la falta de articulación entre las distintas políticas sectoriales (en este caso vivienda e infraestructura de servicios). La falta de cobertura de ambos servicios implica además una baja calidad de vida para las familias localizadas en gran parte de la periferia del partido.

2) Equipamientos de Salud y Educación

La espacialización de los Casos y de los equipamientos muestra que hay una dotación de equipamientos que sirve a una población distribuida de forma irregular en una superficie más o menos extensa en el territorio. Pero al analizar la localización y cobertura de ambos equipamientos se verifica que, tanto en las mejores como en las peores situaciones, hay una gran coincidencia (un poco más deficitaria la cobertura educativa); pero, respecto de cada línea, se encuentran mayores déficit en la línea compra de terreno y construcción, que en la línea construcción.

Se entiende que la accesibilidad a los equipamientos públicos, medidos por la distancia física a ellos, debiera ser equitativa para todos. Pero los resultados demuestran que no es así para todos los casos, creándose en consecuencia desigualdades física-territoriales respecto a su distribución.

3) Centralidades o subcentralidades urbanas

Se observa que hay una gran cantidad de registros localizados cercanos a subcentralidades, en particular el Caso 1 muestra mejores resultados que el Caso 2. No obstante, al visualizar la espacialización de los mismo, en el eje Sureste se verifica que ninguno de los registros, de ambas líneas, cuenta con un subcentro urbano cercano a estas localizaciones.

Lo que implica grandes dificultades en el acceso, medidos por la distancia física a ellos, a servicios y actividades de diferentes escalas, entre otras cosas; entendiendo también que los centros urbanos son un punto de referencia y de expresión simbólica de las condiciones de vida de sus habitantes; y que además ofrecen algunas alternativas de empleo.

4) Transporte público

En cuanto a la cobertura del servicio en el Caso 1 se puede decir que gran parte de la población no encuentran mayores limitaciones con la cobertura, a excepción de aquellas familias localizadas en un sector del eje no que corresponde a la delegación de Arturo Seguí.

Donde se encuentran mayores dificultades es en los registros del Caso 2, esto 
conlleva a una gran dificultad en relación a la conectividad de las variables anteriormente analizadas, ya que es en definitiva la que permite que las familias accedan a servicios y equipamientos públicos en general.

\section{c) Eje regulatorio}

El cruce de los registros con la normativa dio como resultado que en el Caso 1 casi la mitad se encuentra en zonas o sectores no destinados al uso residencial. Entre ellos, las mayores tendencias de localización se encuentran en el Área Urbana, no obstante un gran porcentaje de estos ( $20 \%$ del total) se encuentran en sectores de arroyos y bañados. Si bien se encuentran dentro del área urbana no deja de presentarse como una problemática debido a que se asientan en zonas de máximo riesgo de inundación. Si bien el código establece limitaciones al uso, parcelamiento y volumen edilicio, no impide que se pueda construir en dichos sectores.

También existe un porcentaje considerado (17\%) localizados, dentro del Área Rural, en Zonas Rurales Intensivas, desprovistas en muchos casos de infraestructura de servicios y equipamientos sociales pero, en general, cercanas a las principales vías de acceso que vinculan el partido con la región.

Por otra parte, el $12 \%$ de los registros se localizan, dentro del Área Complementaria, en Zonas de Reserva Urbana, que según la normativa esta destinados al ensanche del Área Urbana: "El uso dominante de esta zona es el Rural Intensivo compatible con el uso residencial limitado, estando condicionadas las intervenciones a la consolidación de las zonas del Área Urbana adyacentes"; y en menor medida, dentro de la misma área complementaria, en Corredores de Servicio y
Zonas Industriales Mixtas; y Rurales Extensivas, perteneciente al área rural.

De los registros de la otra línea (Caso 2 ), se puede observar que ninguno de ellos se encuentra dentro del Área Urbana. Como anteriormente se explicó esta línea impulsó, en el P0artido de La Plata, la re-zonificación de tierras, es decir, los cuatro registros que se encuentran en zona de reserva urbana (C/RU del Área Complementaria), los cinco registros que están en la zona rural intensiva y el único registro que se localiza en zona rural extensiva ( $R / R I$ y R/RE del Área Rural), mediante la sanción de la Ordenanza n¹1094/13 pasarán a renombrarse como zonas residenciales de promoción (U/R3 del Área urbana), solamente por la condición de rezonificación de dichas zonas.

En conclusión, tanto los registros del Caso 1 que se localizan en área no urbanas, como aquellos registros que a partir de la rezonificación serán considerados "dentro del área urbana", en su mayoría se encuentran desprovistos de buenas condiciones urbanas y ambientales, ello significa registros localizados en sectores con déficit de infraestructuras de servicios, con escasa conectividad, con déficit de equipamientos y cercanos a los sectores productivos, lo que implica contaminación de algunos recursos e incompatibilidad de usos; todo ello sólo por condición de no pertenecer al área urbana.

La Foto 2 muestra el paisaje obtenido como resultado de la urbanización de suelos destinados al uso agropecuario:

El Cuadro 2 expresa los resultados obtenidos del análisis de la localización del Caso 1; por su parte, el Cuadro 3 muestra los resultados obtenidos del Caso 2. En ambos casos, los resultados están expresados en 


\section{Foto 2 - Resultado de la urbanización de suelos destinados al uso agropecuario}

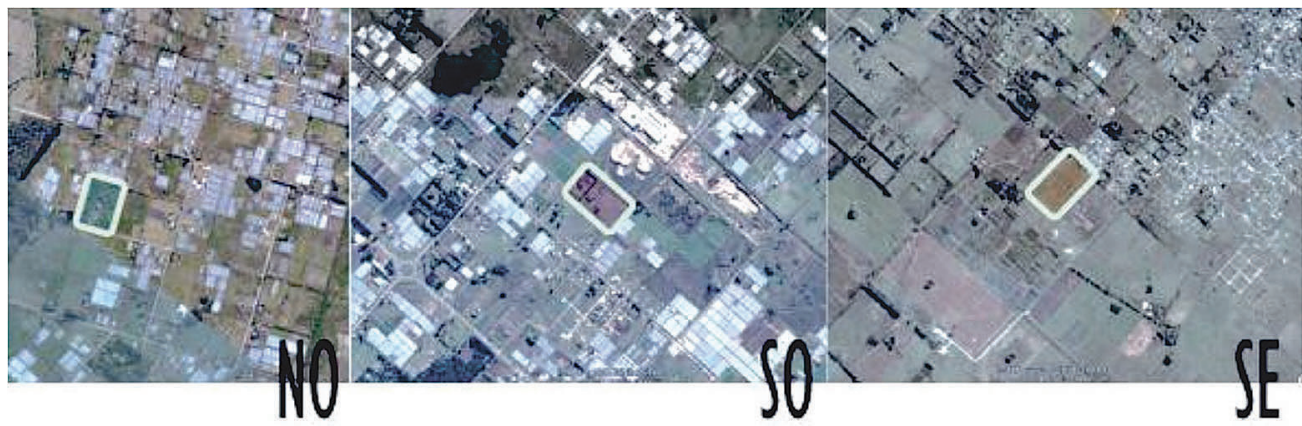

\section{Cuadro 2 - Matriz de análisis: resultados Caso 1}

\begin{tabular}{|c|c|c|c|c|c|c|}
\hline & \multirow{2}{*}{ Eje } & \multirow{2}{*}{ Variable } & \multicolumn{4}{|c|}{ Tratamiento del dato - $\%$} \\
\hline & & & 1 & 2 & 3 & 4 \\
\hline \multirow{7}{*}{ 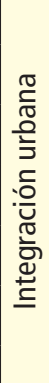 } & Ambiental & Riesgo hídrico & 53,5 & 32,8 & 1,2 & 12,5 \\
\hline & \multirow{5}{*}{ Urbano } & Servicios & 15,1 & 19,4 & 5,8 & 59,7 \\
\hline & & Equipamiento salud & 22,9 & 24,3 & 15,4 & 37,4 \\
\hline & & Equipamiento educativo & 12,5 & 23,8 & 24,9 & 38,8 \\
\hline & & Subcentralidade & 52,2 & 18,8 & 6,7 & 22,3 \\
\hline & & Transporte & 50,1 & 31,0 & 10,1 & 8,8 \\
\hline & Regulatorio & Zonificación de usos de suelo & 69,6 & & 12,8 & 17,7 \\
\hline
\end{tabular}

Fuente: elaboración propia.

Cuadro 3 - Matriz de análisis: resultados Caso 2

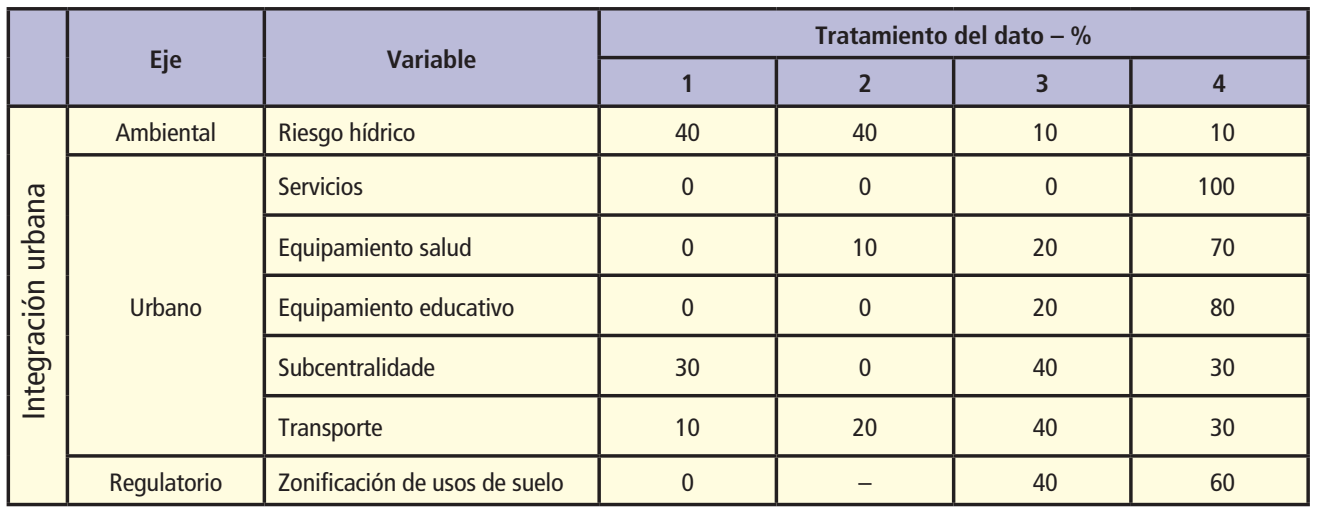

Fuente: elaboración propia. 
porcentaje de registros según se localizan en relación al tratamiento del dato. ${ }^{7}$

\section{Evaluación de la integración urbana}

Una vez analizadas cada una de las variables resulta necesario analizarlas integradamente para poder sintetizar los siguientes puntos:

Identificar los factores que afectan la integración urbana del caso de estudio es necesario para identificar cuáles son los ejes que tienen mayor incidencia en la configuración de las mayores criticidades. El siguiente cuadro representa en porcentajes la síntesis de los tres ejes:

El Cuadro 4 - Síntesis del Caso 1 muestra que el eje que posee el más alto porcentaje de registros está en la escala 1 es el regulatorio, esto significa que si bien no cumple con las mejores condiciones en general es el que muestra mejores resultados en relación a la integración urbana.

Siguiendo en la observación del cuadro, se puede verificar que el eje ambiental muestra los mayores porcentajes de localizaciones en la escala 1, esto significa que a pesar de tener relevancia el hecho de que haya registros localizados en áreas con riesgo ambiental, la mayor cantidad de registros están en una situación favorable.

Por último, en el mismo cuadro, se puede ver que el eje que muestra el mayor porcentaje de registros en la escala 4 de la matriz es el eje urbano. Es decir que los principales factores que afectan a la integración urbana son aquellos que hacen referencia a la distribución de bienes y servicios.

En cambio, en el Cuadro 5 - Síntesis del Caso 2, el mismo eje que anteriormente era el que mejores resultados arrojaba, en este caso es el que presenta los peores valores en la escala 1; mostrando los valores más altos en las escalas 3 y 4 . Esto quiere decir que esta operatoria encuentra al eje regulatorio como uno de los mayores conflictos emergente del Programa en la operatoria donde la adquisición del suelo es un factor determinante.

\section{Cuadro 4 - Síntesis Caso 1}

\begin{tabular}{|l|c|c|c|c|}
\hline Ambiental & $52 \%$ & $35 \%$ & $1 \%$ & $13 \%$ \\
Urbano & $29 \%$ & $24 \%$ & $13 \%$ & $34 \%$ \\
Regulatorio & $59 \%$ & $11 \%$ & $15 \%$ & $15 \%$ \\
\hline
\end{tabular}

\section{Cuadro 5 - Síntesis Caso 2}

\begin{tabular}{|l|c|c|c|c|}
\hline Ambiental & $40 \%$ & $40 \%$ & $10 \%$ & $10 \%$ \\
Urbano & $8 \%$ & $6 \%$ & $24 \%$ & $62 \%$ \\
Regulatorio & $0 \%$ & $26 \%$ & $37 \%$ & $37 \%$ \\
\hline
\end{tabular}


Casi en la misma línea se encuentran los valores del eje urbano, que muestran sus porcentajes más bajos de localizaciones en los niveles 1 y 2 , y los más altos en el 3 y 4. Ello implica que este eje se presenta como el más crítico en relación a las condiciones de integración urbana.

Por último, podría decirse que los mejores resultados son aquellos vinculados al eje ambiental, con los porcentajes de localizaciones más altos en la escala 1 y 2 , que significan casi el total de los registros, teniendo muy poca incidencia las escalas 3 y 4 .
Para concluir, viendo la síntesis de cada operatoria se puede decir que:

- En el eje ambiental las localizaciones no presenta grandes conflictos en ninguna de las dos operatorias. Aunque sería importante que no hubiera porcentajes en los niveles 3 y 4 por el riesgo que conlleva la localización de familias en estos sectores.

- En el eje urbano se presenta como uno de los principales factores que afectan a la integración de las localizaciones con su entorno urbano. Si bien en muchos casos se trata de decisión política para revertir estos

Foto 3 - Síntesis de las operatorias del Caso 1 y 2

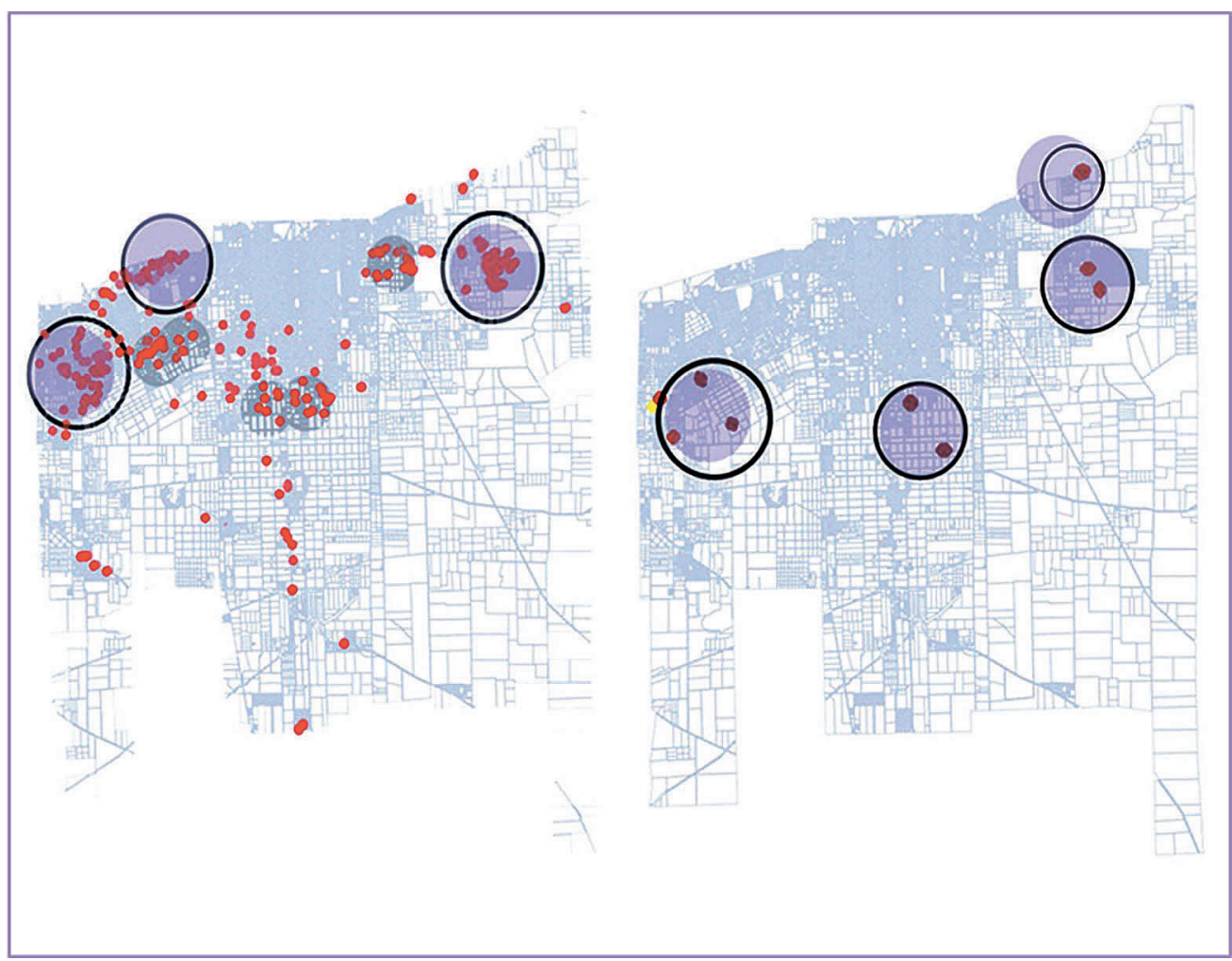

Fuente: elaboración propia (2014). 
conflictos, hoy en día se presenta como una gran problemática porque el conjunto de estas variables hacen a la calidad de vida de la población.

- En el eje regulatorio las localizaciones del Caso 1 no presenta muchos inconvenientes, pero en el Caso 2 sí. El hecho de que haya tanta diferencia entre las dos líneas evidencia las dificultades que tiene el estado municipal para abordar la gestión del suelo urbano.

La identificación de los sectores más críticos del partido se realizó a partir de la evaluación de las distintas variables interrelacionadas. Como se explicó anteriormente en la metodología, en este plano síntesis se espacializarán todas aquellas localizaciones que cuenten con al menos tres variables en la escala de 3 y 4 de la matriz para evaluar si el conjunto de las mismas definen sectores dentro del partido.

El plano síntesis del Caso 1, muestra claramente que se definen tres sectores bien críticos, la intensidad del color muestra mayor cantidad de variables en la escala de 3 y 4 por cada localización. Estos tres sectores, diferenciados del resto, corresponden dos a las periferias del ejes noroeste, precisamente a las delegaciones de Arturo Seguí/Melchor Romero, y Ringuelet/Gonnet, y sector restante corresponde al eje sureste ubicado en la delegación de Villa Elvira.

Por otra parte, pero en menor medida, se identifican conflictivos algunos sectores del eje sudoeste, localizado en el los barrios de San Carlos/Los Hornos, y otro en el eje noroeste, ubicado en los barrios de Gorina/Hernandez. La gran cantidad de registros en el plano muestra que la mayoría de ellos presentan variables en condiciones problemáticas.
Por último, el plano síntesis del Caso 2, muestra claramente que, a excepción de un solo registro localizado en la delegación de Arturo Seguí, todos los registros restantes (9 de 10) tienen al menos 3 variables en la escala 3 y 4 de la matriz, por cada registro. Esto significa que para esta operatoria, los sectores críticos son todos.

\section{Conclusiones}

Como resultado de la exploración, se pudo ver, en primera instancia, que los procesos de expansión urbana han generado cambios en la configuración territorial, acrecentando los débiles límites entre lo urbano y lo rural. Estos proceso, configuraron dos territorios bien diferenciados: por un lado el Casco Urbano, sumado a algunos sectores acotados del eje noroeste y sudoeste, caracterizado por tener un diseño más ordenado y por contar con los servicios básicos para casi el total de su población; y por otro lado, la periferia en donde la expansión se desarrolló sin normas clara que regulen la producción del suelo. En este sentido, la normativa como instrumento de la política urbana, resultó insuficiente para gestionar el territorio.

La zonificación como instrumento debería ser el claro reflejo del reconocimiento de las tendencias que se observan en el territorio, dimensionadas y evaluadas integralmente en cuanto a sus repercusiones territoriales, desde lo social, económico, ambiental, espacial y funcional. Para reducir las marcadas diferencias entre la población que puede llegar a acceder y los que no lo logran. Las desigualdades sociales que se han profundizado como producto de la 
falta de integración entre distintas políticas generan sectores diferenciales dentro de las ciudades.

La implementación del PRO.CRE.AR debería garantizar, paralelamente al acceso a la tierra, una adecuada integración entre calidad urbana y ambiental. Para ello el municipio, en el marco de la Ley, cuenta con mecanismos de gestión que posibilitan al estado asumir el rol de promotor de la producción del suelo urbano y redistribuir los beneficios de la urbanización.

Ahora bien, pareciera necesario reforzar la necesidad de que la política para la vivienda se ajuste a la diversidad de territorios. Las características y problemáticas de las ciudades metropolitanas requieren un manejo específico en cuanto a escala de las demandas, a la provisión de suelo y los valores inmobiliarios.

Asimismo, reforzar la necesidad que la política, en general, sea coordinada entre los tres niveles del Estado, ya que una mala implementación local puede resultar negativa tanto para el buen desarrollo de la iniciativa como para el desarrollo urbano y territorial municipal. $Y$ en particular, reforzar la necesidad de que la política habitacional sea abordada de manera articulada con otras políticas para brindar el acceso al suelo urbanizado, y conducir el crecimiento urbano de manera sustentable, asegurando la integración física, espacial y social de la población.

Para finalizar, interesa destacar que el desarrollo del estudio permitió reconocer los factores que afectan a la integración urbana de cada una de las operatorias analizadas, que han permitido concluir que uno de los factores de peso significativo es la localización de los mismos en áreas no urbanas, pero también aquellas áreas que, aun siendo urbanas, tienen grandes déficit de servicios y equipamientos. Lo mencionado anteriormente evidencia las dificultades que tiene el Estado municipal para gestionar el territorio en general, y brindar el acceso a suelo en condiciones urbanas de calidad, en una ciudad que se expande insustentablemente y donde el problema de la tierra es constante.

\section{María Eugenia Rodríguez Daneri}

Universidad Nacional de La Plata, Facultad de Arquitectura y Urbanismo, Centro de Investigaciones Urbanas y Territoriales. La Plata, Buenos Aires, Argentina.

eugenia.rd@hotmail.com 


\section{Notas}

(1) Desarrollada en el marco de una Becas Interna de Entrenamiento en Investigación de la FAUUNLP. Dirigida por la Arq. Licia Ríos y codirigida por el Arq. Alejandro Lancioni.

(2) Se toma para el análisis todos los centros públicos de salud.

(3) Se considera equipamiento educativo a todos aquellas instituciones de gestión pública inicial, primaria y secundaria.

(4) Ordenanza 10.703 del año 2010

(5) Para el análisis de la cobertura de transporte se tiene en cuenta solo el recorrido del colectivo y del ferrocarril, no las paradas y estaciones.

(6) Recopilados de documentos obrantes de la Dirección de Obras Particulares.

(7) El tratamiento del dato y las escalas se explican en el apartado "Metodología".

\section{Referências}

AGUILAR, A. G. (2002). Las mega-ciudades y las periferias expandidas. Ampliando el concepto en Ciudad de México. EURE. Santiago, v. 28, n. 85, pp. 121-149. Disponível em: http://www.scielo. cl/scielo.php?script=sci_arttext\&pid=S0250-71612002008500007\&lng=es\&tlng=es.10.4067/ S0250-71612002008500007. Acesso em: 7 jul 2014.

(2006). Introducción en el libro Las grandes aglomeraciones y su periferia regional. Experiencias en Latinoamérica y España. México, H. Camara de Diputados/Universidad Nacional Autónoma de México/Consejo Nacional de Ciencia y Tecnología.

AZUELA, A. (2007). “Capítulo Introductorio”. SMOLKA, M. O. y MULLAHY, L. Tendencias y perspectivas de las políticas de suelo. Cambridge, Lincoln Institute of Land Policy.

BAER, L. (2008). Mercado de suelo y acceso a la vivienda en Buenos Aires luego de la crisis de 2001/02. Ciudad y territorio. Estudios Territoriales. Argentina, v. XL, n. 156.

CICCOLELLA, P. (1999). Globalización y dualización en la Región Metropolitana de Buenos Aires. Grandes inversiones y reestructuración socioterritorial en los años noventa. Eure, v. 25, n. 76, pp. 5-27.

CLICHEVSKY, N. (2003). Pobreza y acceso al suelo urbano. Algunas interrogantes sobre las políticas de regularización en América Latina. Santiago de Chile, Cepal.

DEL RíO, J. P. (2008). El acceso al suelo en la política habitacional en el área metropolitana de Buenos Aires. Notas metodológicas sobre un objeto problemático. X JORNADAS DE INVESTIGACIÓN DEL CENTRO DE INVESTIGACIONES GEOGRÁFICAS Y DEL DEPARTAMENTO DE GEOGRAFÍA. Memoria Académica. Argentina.

(2011). Política de vivienda y acceso a la ciudad. Las tierras y los proyectos urbanos en el conurbano bonaerense. XXVII CONGRESO DE LA ASOCIACIÓN LATINOAMERICANA DE SOCIOLOGÍA-UBA. Argentina. 
DE MATTOS, C. A. (1999). Santiago de Chile, Globalización y Expansión Metropolitana: lo que existía sigue existiendo. Eure. Santiago de Chile, v. XXV, n. 77, pp. 29-56.

FERNÁNDEZ WAGNER, R. (2006). Interrogantes sobre la sustentabilidad de la política habitacional Argentina. In: SEMINARIO IBEROAMERICANO DE CIENCIA Y TECNOLOGIA PARA EL HABITAT POPULAR. Construcción y Participación del conocimiento. Córdoba.

FREDIANI, J. (2010). Lógicas y tendencias de la expansión residencial en áreas periurbanas. El Partido de La Plata, Buenos Aires, Argentina, entre 1990 y 2010. Tese de Doutorado. Argentina, Universidad Nacional de La Plata.

LANCIONI, A.; RÍOS, L. y DELACHAUX, A. (2012). Expansión urbana en municipios bonaerenses: alcance y vínculos con las políticas de tierra y vivienda. Jornadas AUGM. Argentina.

LUNGO, M. (2007). Expansión urbana y regulación del uso del suelo en América Latina. San Salvador.

MORALES-SCHECHINGER, C. (2005). Notas sobre la regulación del mercado de suelo y sus instrumentos. México.

ROCCA, M. J. et al. (2010). Procesos de expansión urbana: políticas territoriales y transformaciones emergentes: los casos de Berazategui, Bahía Blanca, Tandil y Trenque Lauquen. Argentina, Universidad Nacional de La Plata.

ROCCA, M. J. y RíOS, L. (2010). Procesos de expansión urbana. Ordenamiento Territorial en el Partido de La Plata. Argentina, Colegio de Arquitectos de la Provincia de Buenos Aires.

SABATINI, F. y BRAIN, I. (2008). La segregación, los guetos y la integración social urbana: mitos y claves. Eure, v. XXXIV, n. 103, pp. 5-26.

SHLOMO, A.; PARENT, J.; CIVCO, D. L. y BLEI, A. (2012). Chapter 1 de Atlas of Urban Expansion. Cambridge/Massachusetts, Lincoln Institute of Land Policy.

VALDÉS, E. (2001). Los guetos urbanos residenciales. El caso del Country Las Delicias. Córdoba, Anuario de la Escuela de Historia, año 1, n. 1, Ed. Ferreira.

VIDAL ROJAS, R. (1997). Metrópolis en recomposición: elementos para una teoría de la fragmentación urbana. $6^{\circ}$ ENCUENTRO DE GEOGRAFÍA DE AMÉRICA LATINA, BUENOS AIRES. Argentina.

Texto recebido em 31/jmaio/2015

Texto aprovado em 13/nov/2015 